\title{
THE PARTITION ALGEBRA AND THE KRONECKER COEFFICIENTS
}

\author{
C. BOWMAN, M. DE VISSCHER, AND R. ORELLANA
}

\begin{abstract}
We propose a new approach to study the Kronecker coefficients by using the Schur-Weyl duality between the symmetric group and the partition algebra. We explain the limiting behaviour and associated bounds in the context of the partition algebra. Our analysis leads to a uniform description of the reduced Kronecker coefficients when one of the indexing partitions is a hook or a two-part partition.
\end{abstract}

\section{INTRODUCTION}

A fundamental problem in the representation theory of the symmetric group is to describe the coefficients in the decomposition of the tensor product of two Specht modules. These coefficients are known in the literature as the Kronecker coefficients. They are labelled by triples of partitions. Finding a formula or combinatorial interpretation for these coefficients has been described by Richard Stanley as 'one of the main problems in the combinatorial representation theory of the symmetric group'. This question has received the attention of Littlewood [Lit58, James [JK81, Chapter 2.9], Lascoux [Las80], Thibon [Thi91], Garsia and Remmel GR85, Kleshchev and Bessenrodt BK99 amongst others, and yet a combinatorial solution has remained beyond reach for over a hundred years.

Murnaghan discovered an amazing limiting phenomenon satisfied by the Kronecker coefficients; as we increase the length of the first row of the indexing partitions the sequence of Kronecker coefficients obtained stabilises. The limits of these sequences are known as the reduced Kronecker coefficients.

The novel idea of this paper is to study the Kronecker and reduced Kronecker coefficients through the Schur-Weyl duality between the symmetric group, $\mathfrak{S}_{n}$, and the partition algebra, $P_{r}(n)$. The key observation being that the tensor product of Specht modules corresponds to the restriction of simple modules in $P_{r}(n)$ to a Young subalgebra. The combinatorics underlying the representation theory of both objects is based on partitions. The Schur-Weyl duality results in a functor, $\mathrm{F}: \mathfrak{S}_{n}$-mod $\rightarrow P_{r}(n)$-mod, which acts by first row removal on the partitions labelling the simple modules. We exploit this functor along with the following three key facts concerning the representation theory of the partition algebra: (a) it is semisimple for large $n$; (b) it has a stratification by symmetric groups; (c) its non-semisimple representation theory is well developed.

We interpret the Kronecker and reduced Kronecker coefficients and the passage between them in terms of the representation theory of the partition algebra. The limiting phenomenon discovered by Murnaghan and some associated bounds (due

Received by the editors March 6, 2013 and, in revised form, June 10, 2013 and July 4, 2013. 2010 Mathematics Subject Classification. Primary 20C30, 05E10. 
to Brion) are then naturally explained by the fact that $P_{r}(n)$ is semisimple for large enough $n$.

Using the partition algebra we give a simple, unified approach to the cases where one of the indexing partitions is a hook or a two-part partition. In particular, we obtain a positive formula in terms of the Littlewood-Richardson coefficients for the reduced Kronecker coefficients in these cases (see Corollary 5.1). These two cases have already been studied separately - a combinatorial interpretation has been given for all Kronecker coefficients in the single hook case in Bla12, and a formula in terms of Littlewood-Richardson coefficients has been given for the reduced coefficients in the single two-part partition case in [BO05].

Our approach brings forward a general tool to study these coefficients and provides a natural framework for the study of the outstanding problems in the area. In particular, one should notice that our proofs are surprisingly elementary.

The paper is organised as follows. In Sections 1 and 2 we recall the combinatorics underlying the representation theories of the symmetric group and partition algebra. In Section 3 we show how to pass the Kronecker problem through Schur-Weyl duality and phrase it as a question concerning the partition algebra. We then summarise results concerning the Kronecker and reduced Kronecker coefficients that have a natural interpretation in this setting. Section 4 contains a description of the restriction of a standard module for $P_{r}(n)$ to a Young subalgebra, giving a new representation theoretic interpretation of [BOR11, Lemma 2.1]. In Section 5 we specialise to hook and two-part partitions and obtain positive formulas for the reduced Kronecker coefficients in these cases. Section 6 contains an extended example.

\section{SymmetriC GROUP COMBINATORICS}

The combinatorics underlying the representation theory of the symmetric group, $\mathfrak{S}_{n}$, is based on partitions. A partition $\lambda$ of $n$, denoted $\lambda \vdash n$, is defined to be a weakly decreasing sequence $\lambda=\left(\lambda_{1}, \lambda_{2}, \ldots, \lambda_{\ell}\right)$ of non-negative integers such that the sum $|\lambda|=\lambda_{1}+\lambda_{2}+\cdots+\lambda_{\ell}$ equals $n$. The length of a partition is the number of non-zero parts; we denote this by $\ell(\lambda)$. We let $\Lambda_{n}$ denote the set of all partitions of $n$.

With a partition, $\lambda$, is associated its Young diagram, which is the set of nodes

$$
[\lambda]=\left\{(i, j) \in \mathbb{Z}_{>0}^{2} \mid j \leq \lambda_{i}\right\} .
$$

Given a node specified by $i, j \geq 1$, we say the node has content $j-i$. We let $\operatorname{ct}\left(\lambda_{i}\right)$ denote the content of the last node in the $i$ th row of $[\lambda]$, that is, $\operatorname{ct}\left(\lambda_{i}\right)=\lambda_{i}-i$.

Over the complex numbers, the irreducible Specht modules, $\mathbf{S}(\lambda)$, of $\mathfrak{S}_{n}$ are indexed by the partitions, $\lambda$, of $n$. An explicit construction of these modules is given in JK81.

1.1. The classical Littlewood-Richardson rule. The Littlewood-Richardson rule is a combinatorial description of the restriction of a Specht module to a Young subgroup of the symmetric group. Through Schur-Weyl duality, the rule also computes the decomposition of a tensor product of two simple modules of $\mathrm{GL}_{n}(\mathbb{C})$. The Littlewood-Richardson rule is the most famous algorithm for decomposing tensor products and has been generalised in several directions.

The following is a simple restatement of this rule as it appears in JK81, Section 2.8.13]. 
Theorem 1.1 (The Littlewood-Richardson Rule). For $\lambda \vdash r_{1}, \mu \vdash r_{2}$ and $\nu \vdash$ $r_{1}+r_{2}$,

$$
\mathbf{S}(\nu) \downarrow_{\mathfrak{S}_{r_{1}} \times \mathfrak{S}_{r_{2}}}^{\mathfrak{S}_{r_{1}+r_{2}}} \cong \bigoplus_{\lambda \vdash r_{1}, \mu \vdash r_{2}} c_{\lambda, \mu}^{\nu} \mathbf{S}(\lambda) \otimes \mathbf{S}(\mu),
$$

where the $c_{\lambda, \mu}^{\nu}$ are the Littlewood-Richardson coefficients (defined below).

The Littlewood-Richardson coefficient $c_{\lambda, \mu}^{\nu}$ is zero, unless $\lambda \subseteq \nu$, and, otherwise may be calculated as follows. For each node $(i, j)$ of $\mu$, take a symbol $u_{i, j}$. Begin with the diagram $\lambda$ and:

(1) Add to it all symbols $u_{1, j}$ (corresponding to the first row of nodes of $\mu$ ) in such a way as to produce the diagram of a partition and to satisfy (3).

(2) Next add all symbols $u_{2, j}$ (corresponding to the second row of nodes of $\mu$ ) following the same rules. Continue this process with all rows of $\mu$.

(3) The added symbols must satisfy: (a) for all $i$, if $y<j$, then $u_{i, y}$ appears in a later column than $u_{i, j}$; and (b) for all $j$, if $x<i$, then $u_{x, j}$ is in an earlier row than $u_{i, j}$.

By the transitivity of induction the Littlewood-Richardson rule determines the structure of the restriction of a Specht module to any Young subgroup. Of particular importance in this paper is the three-part case

$$
\begin{aligned}
& \mathbf{S}(\nu) \downarrow_{\mathfrak{S}_{r_{1}} \times \mathfrak{S}_{r_{2}} \times \mathfrak{S}_{r_{3}}}^{\mathfrak{S}_{r_{1}+r_{2}+r_{3}}} \cong \bigoplus_{\substack{\xi \vdash r_{1}+r_{2} \\
\nu \vdash r_{3}}}\left(c_{\xi, \eta}^{\nu} \mathbf{S}(\xi) \otimes \mathbf{S}(\eta)\right) \downarrow_{\mathfrak{S}_{r_{1}} \times \mathfrak{S}_{r_{2}} \times \mathfrak{S}_{r_{3}}}^{\mathfrak{S}_{r_{1}+r_{2}} \times \mathfrak{S}_{r_{3}}} \\
& \cong \bigoplus_{\substack{\lambda \vdash r_{1}, \mu \vdash r_{2}, \nu \vdash r_{3}}}\left(\sum_{\xi \vdash r_{1}+r_{2}} c_{\lambda, \mu}^{\xi} c_{\xi, \eta}^{\nu}\right) \mathbf{S}(\lambda) \otimes \mathbf{S}(\mu) \otimes \mathbf{S}(\eta)
\end{aligned}
$$

We therefore set $c_{\lambda, \mu, \eta}^{\nu}=\sum_{\xi} c_{\lambda, \mu}^{\xi} c_{\xi, \eta}^{\nu}$.

1.2. Tensor products of Specht modules of the symmetric group. In this section we define the Kronecker coefficients and the reduced Kronecker coefficients as well as set some notation. Let $\lambda$ and $\mu$ be two partitions of $n$. Then

$$
\mathbf{S}(\lambda) \otimes \mathbf{S}(\mu)=\bigoplus_{\nu \vdash n} g_{\lambda, \mu}^{\nu} \mathbf{S}(\nu) ;
$$

the coefficients $g_{\lambda, \mu}^{\nu}$ are known as the Kronecker coefficients. These coefficients satisfy an amazing stability property illustrated in the following example.

Example 1.2. We have the following tensor products of Specht modules:

$$
\begin{aligned}
\mathbf{S}\left(1^{2}\right) \otimes \mathbf{S}\left(1^{2}\right) & =\mathbf{S}(2), \\
\mathbf{S}(2,1) \otimes \mathbf{S}(2,1) & =\mathbf{S}(3) \oplus \mathbf{S}(2,1) \oplus \mathbf{S}\left(1^{3}\right), \\
\mathbf{S}(3,1) \otimes \mathbf{S}(3,1) & =\mathbf{S}(4) \oplus \mathbf{S}(3,1) \oplus \mathbf{S}\left(2,1^{2}\right) \oplus \mathbf{S}\left(2^{2}\right)
\end{aligned}
$$

at which point the product stabilises, i.e. for all $n \geq 4$, we have

$$
\mathbf{S}(n-1,1) \otimes \mathbf{S}(n-1,1)=\mathbf{S}(n) \oplus \mathbf{S}(n-1,1) \oplus \mathbf{S}\left(n-2,1^{2}\right) \oplus \mathbf{S}(n-2,2) .
$$


Let $\lambda=\left(\lambda_{1}, \lambda_{2}, \ldots, \lambda_{\ell}\right)$ be a partition and $n$ be an integer, and define $\lambda_{[n]}=$ $\left(n-|\lambda|, \lambda_{1}, \lambda_{2}, \ldots, \lambda_{\ell}\right)$. Note that all partitions of $n$ can be written in this form.

For $\lambda_{[n]}, \mu_{[n]}, \nu_{[n]} \in \Lambda_{n}$ we let

$$
g_{\lambda_{[n]}, \mu_{[n]}}^{\nu_{[n]}}=\operatorname{dim}_{\mathbb{C}}\left(\operatorname{Hom}_{\mathfrak{S}_{n}}\left(\mathbf{S}\left(\lambda_{[n]}\right) \otimes \mathbf{S}\left(\mu_{[n]}\right), \mathbf{S}\left(\nu_{[n]}\right)\right)\right)
$$

denote the multiplicity of $\mathbf{S}\left(\nu_{[n]}\right)$ in the tensor product $\mathbf{S}\left(\lambda_{[n]}\right) \otimes \mathbf{S}\left(\mu_{[n]}\right)$. Murnaghan showed (see [Mur38, Mur55]) that if we allow the first parts of the partitions to increase in length, then we obtain a limiting behaviour as follows. For $\lambda_{[N]}, \mu_{[N]}, \nu_{[N]} \in \Lambda_{N}$ and $N$ sufficiently large we have that

$$
g_{\lambda_{[N+k]}, \mu_{[N+k]}}^{\nu_{[N+k]}}=\bar{g}_{\lambda, \mu}^{\nu}
$$

for all $k \geq 1$; the integers $\bar{g}_{\lambda, \mu}^{\nu}$ are called the reduced Kronecker coefficients. Bounds for this stability have been given in [Bri93, Val99, Kly04, BOR11].

Remark 1.3. The reduced Kronecker coefficients are also the structural constants for a linear basis for the polynomials in countably many variables known as the character polynomials; see [Mac95].

\section{The partition Algebra}

The partition algebra was originally defined by Martin in Mar91. All the results in this section are due to Martin and his collaborators; see Mar96] and the references therein.

2.1. Definitions. For $r \in \mathbb{Z}_{>0}, \delta \in \mathbb{C}$, we let $P_{r}(\delta)$ denote the complex vector space with basis given by all set-partitions of $\{1,2, \ldots, r, \overline{1}, \overline{2}, \ldots, \bar{r}\}$. A part of a set-partition is called a block. For example,

$$
d=\{\{1,2,4, \overline{2}, \overline{5}\},\{3\},\{5,6,7, \overline{3}, \overline{4}, \overline{6}, \overline{7}\},\{8, \overline{8}\},\{\overline{1}\}\}
$$

is a set-partition (for $r=8$ ) with 5 blocks.

A set-partition can be represented by an $(r, r)$-partition diagram consisting of a frame with $r$ distinguished points on the northern and southern boundaries, which we call vertices. We number the northern vertices from left to right by $1,2, \ldots, r$ and the southern vertices similarly by $\overline{1}, \overline{2}, \ldots, \bar{r}$ and connect two vertices by a path if they belong to the same block. Note that such a diagram is not uniquely defined; two diagrams representing the set-partition $d$ above are given in Figure 1 .
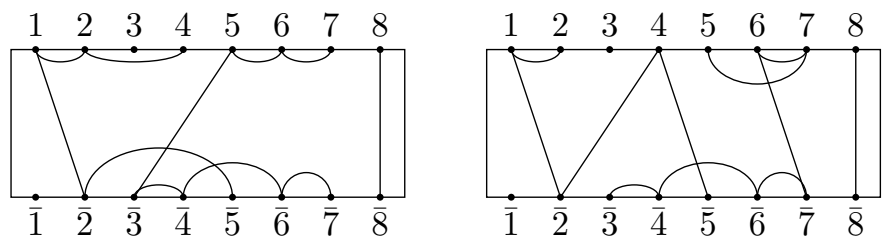

Figure 1. Two representatives of the set-partition $d$.

We can generalise this definition to $(r, m)$-partition diagrams as diagrams representing set-partitions of $\{1, \ldots, r, \overline{1}, \ldots, \bar{m}\}$ in the obvious way.

We define the product $x \cdot y$ of two diagrams $x$ and $y$ using the concatenation of $x$ above $y$, where we identify the southern vertices of $x$ with the northern vertices of $y$. If there are $t$ connected components consisting only of middle vertices, then the 
product is set equal to $\delta^{t}$ times the diagram with the middle components removed. Extending this by linearity defines a multiplication on $P_{r}(\delta)$.

We now fix a unique choice of $(r, r)$-partition diagram corresponding to a given set-partition. Note that any block in a set-partition is of the form $A \cup B$ where $A=\left\{i_{1}<i_{2}<\ldots<i_{p}\right\}$ and $B=\left\{\bar{j}_{1}<\overline{j_{2}}<\ldots<\bar{j}_{q}\right\}$ (and $A$ or $B$ could be empty). We draw this block by putting an arc joining each pair $\left(i_{l}, i_{l+1}\right)$ and $\left(\bar{j}_{l}, \bar{j}_{l+1}\right)$, and if $A$ and $B$ are non-empty we draw a strand from $i_{1}$ to $\bar{j}_{1}$, that is, we draw a single propagating line on the leftmost vertices of the block. Blocks containing a northern and a southern vertex will be called propagating blocks; all other blocks will be called non-propagating blocks. Note that in Figure 1, the leftmost diagram is of this form. We often omit the numbering on the vertices.

It is known that $P_{r}(\delta)$ is generated by the elements $\mathrm{s}_{i, j}, \mathrm{p}_{i, j}(1 \leq i<j \leq r)$ and $\mathrm{p}_{i}(1 \leq i \leq r)$ depicted in Figure 2 below (see [Mar96, Proposition 1]).
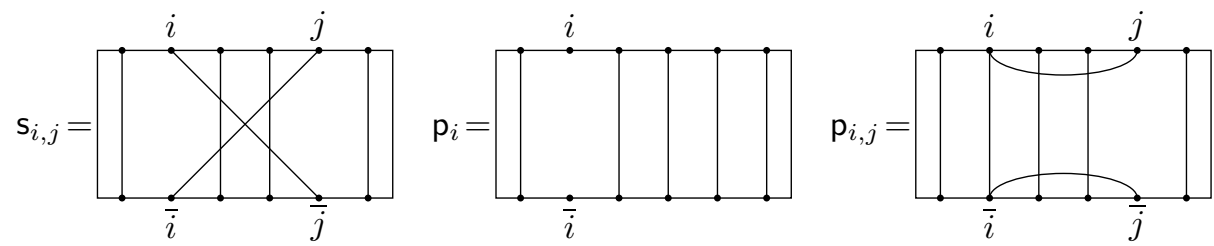

Figure 2. Generators of $P_{r}(n)$.

Assumption. We assume throughout the paper that $\delta \neq 0$.

2.2. Filtration by propagating blocks and standard modules. Fix $\delta \in \mathbb{C}^{\times}$ and write $P_{r}=P_{r}(\delta)$. Note that the multiplication in $P_{r}$ cannot increase the number of propagating blocks. More precisely, if $x$, respectively $y$, is a partition diagram with $p_{x}$, respectively $p_{y}$, propagating blocks, then $x \cdot y$ is equal to $\delta^{t} z$ for some $t \geq 0$ and some partition diagram $z$ with $p_{z}$ propagating blocks, where $p_{z} \leq$ $\min \left\{p_{x}, p_{y}\right\}$. This gives a filtration of the algebra $P_{r}$ by the number of propagating blocks. This filtration can be realised using the idempotents $\mathrm{e}_{l}=\delta^{-l} \mathrm{p}_{1} \mathrm{p}_{2} \ldots \mathrm{p}_{l}$ $(1 \leq l \leq r)$, depicted in Figure 3

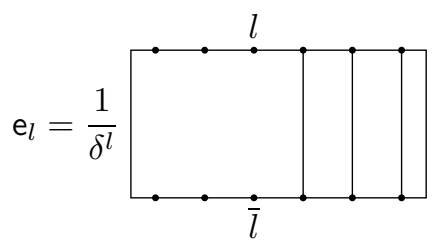

FiguRE 3. The idempotent $\mathrm{e}_{l}$.

We have

$$
P_{r} \mathrm{e}_{r} P_{r} \subset P_{r} \mathrm{e}_{r-1} P_{r} \subset \ldots \subset P_{r} \mathrm{e}_{1} P_{r} \subset P_{r} .
$$

It is easy to see that

$$
\mathrm{e}_{1} P_{r} \mathrm{e}_{1} \cong P_{r-1}
$$


and that this generalises to $P_{r-l} \cong \mathrm{e}_{l} P_{r} \mathrm{e}_{l}$ for $1 \leq l \leq r$. Moreover, $P_{r} \mathrm{e}_{1} P_{r}$ is the span of all $(r, r)$-partition diagrams with at most $r-1$ propagating blocks, and hence we have

$$
P_{r} /\left(P_{r} \mathrm{e}_{1} P_{r}\right) \cong \mathbb{C S}_{r} .
$$

Using equation (2.2), we get that any $\mathbb{C S}_{r}$-module can be inflated to a $P_{r}$-module. We also get from equations (2.1) and (2.2), by induction, that the simple $P_{r}$-modules are indexed by the set $\Lambda_{\leq r}=\bigcup_{0<i<r} \Lambda_{i}$.

For any $\nu \in \Lambda_{\leq r}$ with $\nu \vdash r-\bar{l}$, we define a $P_{r}$-module, $\Delta_{r}(\nu)$, by

$$
\Delta_{r}(\nu)=P_{r} \mathrm{e}_{l} \otimes_{P_{r-l}} \mathbf{S}(\nu) .
$$

(Here we have identified $P_{r-l}$ with $\mathrm{e}_{l} P_{r} \mathrm{e}_{l}$ using the isomorphism given in equation (2.1), and the action of $P_{r}$ on $\Delta_{r}(\nu)$ is given by left multiplication.) These modules are easily seen to coincide with the modules defined in [Mar96, Definition 7].

It is known ([MS93] ) that $P_{r}(\delta)$ is semisimple if and only if $\delta \notin\{0,1, \ldots, 2 r-2\}$, and in this case the set $\left\{\Delta_{r}(\nu): \nu \in \Lambda_{\leq r}\right\}$ forms a complete set of non-isomorphic simple modules.

In general, the algebra $P_{r}(\delta)$ is quasi-hereditary with respect to the partial order on $\Lambda_{\leq r}$ given by $\lambda<\mu$ if $|\lambda|>|\mu|$ (see [Mar96, Proposition 3]). The modules $\Delta_{r}(\nu)$ are the standard modules, each of which has a simple head $L_{r}(\nu)$, and the set $\left\{L_{r}(\nu): \nu \in \Lambda_{\leq r}\right\}$ forms a complete set of non-isomorphic simple modules.

We now give an explicit description of the standard modules which follows directly from (2.3). We set $V(r, r-l)$ to be the span of all $(r, r-l)$-partition diagrams having precisely $(r-l)$ propagating blocks. This has a natural structure of a $\left(P_{r}(\delta), \mathfrak{S}_{r-l}\right)$-bimodule. It is easy to see that, as vector spaces, we have

$$
\Delta_{r}(\nu) \cong V(r, r-l) \otimes_{\mathfrak{S}_{r-l}} \mathbf{S}(\nu) .
$$

The action of $P_{r}(\delta)$ is given as follows. Let $v$ be a partition diagram in $V(r, r-l)$, $x \in \mathbf{S}(\nu)$ and $X$ be an $(r, r)$-partition diagram. Concatenate $X$ and $v$ to get $\delta^{t} v^{\prime}$ for some $(r, r-l)$-partition diagram $v^{\prime}$ and some non-negative integer $t$. If $v^{\prime}$ has fewer than $(r-l)$ propagating blocks, then we set $X(v \otimes x)=0$. Otherwise we set $X(v \otimes x)=\delta^{t} v^{\prime} \otimes x$.

Note that if $\nu \vdash r$, then we have

$$
\Delta_{r}(\nu) \cong V(r, r) \otimes_{\mathfrak{S}_{r}} \mathbf{S}(\nu)=\mathbf{S}(\nu),
$$

viewed as a $P_{r}(\delta)$-module via equation (2.2).

2.3. Non-semisimple representation theory of the partition algebra. We assume that $\delta=n \in \mathbb{Z}_{>0}$ (the algebra is semisimple for $n \notin \mathbb{Z}_{\geq 0}$, and we shall not require the degenerate $n=0$ case).

Definition 2.1 ([Mar96, Section 2]). Let $\mu \subset \lambda$ be partitions. We say that $(\mu, \lambda)$ is an $n$-pair, and write $\mu \hookrightarrow_{n} \lambda$ if the Young diagram of $\lambda$ differs from the Young diagram of $\mu$ by a horizontal row of boxes of which the last (rightmost) one has content $n-|\mu|$.

Example 2.2. For example, $((2,1),(4,1))$ is a 6 -pair. We have that $6-|\mu|=3$ and the Young diagrams (with contents) are as follows:

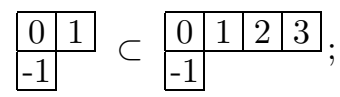

note that they differ by \begin{tabular}{|l|l|}
\hline 2 & 3
\end{tabular} . 
Recall that the set of simple (or standard) modules for $P_{r}(n)$ are labelled by the set $\Lambda_{\leq r}$. This set splits into $P_{r}(n)$-blocks. In [Mar96, Proposition 9], Martin gives a complete description of these blocks and the structure of the standard modules, which we now recall. The set of labels in each block forms a maximal chain of $n$-pairs:

$$
\lambda^{(0)} \hookrightarrow_{n} \lambda^{(1)} \hookrightarrow_{n} \lambda^{(2)} \hookrightarrow_{n} \ldots \hookrightarrow_{n} \lambda^{(t)} .
$$

Moreover, for $1 \leq i \leq t$ we have that $\lambda^{(i)} / \lambda^{(i-1)}$ consists of a strip of boxes in the $i$ th row. Now we have an exact sequence of $P_{r}(n)$-modules:

$$
0 \rightarrow \Delta_{r}\left(\lambda^{(t)}\right) \rightarrow \ldots \rightarrow \Delta_{r}\left(\lambda^{(2)}\right) \rightarrow \Delta_{r}\left(\lambda^{(1)}\right) \rightarrow \Delta_{r}\left(\lambda^{(0)}\right) \rightarrow L_{r}\left(\lambda^{(0)}\right) \rightarrow 0
$$

with the image of each homomorphism being simple. Each standard module $\Delta_{r}\left(\lambda^{(i)}\right)$ (for $0 \leq i \leq t-1$ ) has Loewy structure

$$
\begin{gathered}
L_{r}\left(\lambda^{(i)}\right), \\
L_{r}\left(\lambda^{(i+1)}\right),
\end{gathered}
$$

and so in the Grothendieck group we have

$$
\left[L_{r}\left(\lambda^{(i)}\right)\right]=\sum_{j=i}^{t}(-1)^{j-i}\left[\Delta_{r}\left(\lambda^{(j)}\right)\right] .
$$

Note that each block is totally ordered by the size of the partitions. Note also that it follows from (2.4) that if $\lambda$ is maximal in its block, then $\Delta_{r}(\lambda)=L_{r}(\lambda)$; in particular, if $\lambda$ is a partition of $r$ this is always the case.

Proposition 2.3. Let $\nu \in \Lambda_{\leq r}$ and assume that $\nu_{[n]}$ is a partition. Then we have that

(i) $\nu$ is the minimal element in its $P_{r}(n)$-block, and

(ii) $\nu$ is the unique element in its block if and only if $n+1-\nu_{1}>r$.

Proof. (i) Observe that for $\nu_{[n]}$ to be a partition we must have $n-|\nu| \geq \nu_{1}$. This implies that $\operatorname{ct}\left(\nu_{1}\right)=\nu_{1}-1 \leq n-|\nu|-1$. So we have $\nu \hookrightarrow_{n} \mu$ for some partition $\mu$ with $\mu / \nu$ being a single strip in the first row. Thus we have $\nu=\nu^{(0)}$ and $\mu=\nu^{(1)}$.

(ii) Now as $\nu^{(1)} / \nu$ is a single strip in the first row with last box having content $n-|\nu|$, we have that $\left|\nu^{(1)} / \nu\right|=n-|\nu|+1-\nu_{1}$ and thus $\left|\nu^{(1)}\right|=n+1-\nu_{1}$. Thus $n+1-\nu_{1}>r$ if and only if $\nu^{(1)} \notin \Lambda_{\leq r}$, which is equivalent to $\nu$ being the only partition in its $P_{r}(n)$-block.

\section{SChUR-Weyl DUALity}

Classical Schur-Weyl duality is the relationship between the general linear and symmetric groups over tensor space. To be more specific, let $V_{n}$ be an $n$-dimensional complex vector space and let $V_{n}^{\otimes r}$ denote its $r$ th tensor power.

We have that the symmetric group $\mathfrak{S}_{r}$ acts on the right by permuting the factors. The general linear group, $\mathrm{GL}_{n}$, acts on the left by matrix multiplication on each factor. These two actions commute, and moreover $\mathrm{GL}_{n}$ and $\mathfrak{S}_{r}$ are full mutual centralisers in $\operatorname{End}\left(V_{n}^{\otimes r}\right)$.

The partition algebra, $P_{r}(n)$, plays the role of the symmetric group, $\mathfrak{S}_{r}$, when we restrict the action of $\mathrm{GL}_{n}$ to the subgroup of permutation matrices, $\mathfrak{S}_{n}$. 
3.1. Schur-Weyl duality between $\mathfrak{S}_{n}$ and $P_{r}(n)$. Let $V_{n}$ denote an $n$ dimensional complex space. Then $\mathfrak{S}_{n}$ acts on $V_{n}$ via the permutation matrices

$$
\sigma \cdot v_{i}=v_{\sigma(i)} \quad \text { for } \sigma \in \mathfrak{S}_{n} .
$$

Notice that we are simply restricting the $\mathrm{GL}_{n}$ action in the classical Schur-Weyl duality to the permutation matrices. Thus, $\mathfrak{S}_{n}$ acts diagonally on the basis of simple tensors of $V_{n}^{\otimes r}$ as follows:

$$
\sigma \cdot\left(v_{i_{1}} \otimes v_{i_{2}} \otimes \cdots \otimes v_{i_{r}}\right)=v_{\sigma\left(i_{1}\right)} \otimes v_{\sigma\left(i_{2}\right)} \otimes \cdots \otimes v_{\sigma\left(i_{r}\right)} .
$$

For each $(r, r)$-partition diagram $d$ and each integer sequence $i_{1} \ldots, i_{r}, i_{\overline{1}}, \ldots, i_{\bar{r}}$ with $1 \leq i_{j}, i_{\bar{j}} \leq n$, define

$$
\phi_{r, n}(d)_{i_{\overline{1}}, \ldots, i_{\bar{r}}}^{i_{1}, \ldots, i_{r}}= \begin{cases}1 & \text { if } i_{t}=i_{s} \text { whenever vertices } t \text { and } s \text { are connected in } d, \\ 0 & \text { otherwise. }\end{cases}
$$

A partition diagram $d \in P_{r}(n)$ acts on the basis of simple tensors of $V_{n}^{\otimes r}$ as follows:

$$
\Phi_{r, n}(d)\left(v_{i_{1}} \otimes v_{i_{2}} \otimes \cdots \otimes v_{i_{r}}\right)=\sum_{i_{\overline{1}}, \ldots, i_{\bar{r}}} \phi_{r, n}(d)_{i_{\overline{1}}, \ldots, i_{\bar{r}}}^{i_{1}, \ldots, i_{r}} v_{i_{\overline{1}}} \otimes v_{i_{\overline{2}}} \otimes \cdots \otimes v_{i_{\bar{r}}} .
$$

Theorem 3.1 (Jones Jon94]). $\mathfrak{S}_{n}$ and $P_{r}(n)$ generate the full centralisers of each other in $\operatorname{End}\left(V_{n}^{\otimes r}\right)$.

(a) $P_{r}(n)$ generates $\operatorname{End}_{\mathfrak{S}_{n}}\left(V_{n}^{\otimes r}\right)$, and when $n \geq 2 r, P_{r}(n) \cong \operatorname{End}_{\mathfrak{S}_{n}}\left(V_{n}^{\otimes r}\right)$.

(b) $\mathfrak{S}_{n}$ generates $\operatorname{End}_{P_{r}(n)}\left(V_{n}^{\otimes r}\right)$.

We will denote $E_{r}(n)=\operatorname{End}_{\mathfrak{S}_{n}}\left(V_{n}^{\otimes r}\right)$. Note that as $V_{n}^{\otimes r}$ is a semisimple $\mathbb{C S}_{r^{-}}$ module (as $\mathbb{C S}_{r}$ is a semisimple algebra), we have that $E_{r}(n)$ is a semisimple algebra (see for example [Ben98, Theorem 1.3.4]).

Theorem 3.2 (Mar96]; see also [HR05]). We have a decomposition of $V_{n}^{\otimes r}$ as a $\left(\mathfrak{S}_{n}, P_{r}(n)\right)$-bimodule

$$
V_{n}^{\otimes r}=\bigoplus \mathbf{S}\left(\lambda_{[n]}\right) \otimes L_{r}(\lambda)
$$

where the sum is over all partitions $\lambda_{[n]}$ of $n$ such that $|\lambda| \leq r$.

Using [GW98, Theorem 9.2.2] we have, for $\lambda_{[n]}, \mu_{[n]}, \nu_{[n]} \vdash n$ with $\lambda \vdash r$ and $\mu \vdash s$

$$
\begin{gathered}
\operatorname{Hom}_{\mathfrak{S}_{n}}\left(\mathbf{S}\left(\nu_{[n]}\right), \mathbf{S}\left(\lambda_{[n]}\right) \otimes \mathbf{S}\left(\mu_{[n]}\right)\right) \\
\cong \begin{cases}\operatorname{Hom}_{E_{r}(n) \otimes E_{s}(n)}\left(L_{r}(\lambda) \otimes L_{s}(\mu), L_{r+s}(\nu) \downarrow_{E_{r}(n) \otimes E_{s}(n)}\right) & \text { if } \nu \in \Lambda_{\leq r+s}, \\
0 & \text { otherwise. }\end{cases}
\end{gathered}
$$

Now $L_{r+s}(\nu)$ is a simple $P_{r+s}(n)$-module annihilated by $\operatorname{ker} \Phi_{r+s, n}$ and hence also by $\operatorname{ker} \Phi_{r, n} \otimes \operatorname{ker} \Phi_{s, n}$. Therefore, $L_{r+s}(\nu) \downarrow_{P_{r}(n) \otimes P_{s}(n)}$ is the semisimple $E_{r}(n) \otimes$ $E_{s}(n)$-module $L_{r+s}(\nu) \downarrow_{E_{r}(n) \otimes E_{s}(n)}$ inflated to $P_{r}(n) \otimes P_{s}(n)$, and so

$$
\begin{gathered}
\operatorname{Hom}_{\mathfrak{S}_{n}}\left(\mathbf{S}\left(\nu_{[n]}\right), \mathbf{S}\left(\lambda_{[n]}\right) \otimes \mathbf{S}\left(\mu_{[n]}\right)\right) \\
\cong \begin{cases}\operatorname{Hom}_{P_{r}(n) \otimes P_{s}(n)}\left(L_{r}(\lambda) \otimes L_{s}(\mu), L_{r+s}(\nu) \downarrow_{P_{r}(n) \otimes P_{s}(n)}\right) & \text { if } \nu \in \Lambda_{\leq r+s}, \\
0 & \text { otherwise. }\end{cases}
\end{gathered}
$$

In the next section, we shall implicitly use the fact that $L_{r}(\lambda)=\Delta_{r}(\lambda)$ and $L_{s}(\lambda)=$ $\Delta_{s}(\lambda)$ for $\lambda \vdash r, \mu \vdash s$. 
3.2. Kronecker product via the partition algebra. Combining (3.3) with (2.4) and Proposition 2.3(i) we have the following result.

Theorem 3.3. Let $\lambda_{[n]}, \mu_{[n]}, \nu_{[n]} \vdash n$ with $\lambda \vdash r$ and $\mu \vdash s$. Then we have

$$
g_{\lambda_{[n]}, \mu_{[n]}}^{\nu_{[n]}}= \begin{cases}\sum_{i=0}^{t}(-1)^{i}\left[\Delta_{r+s}\left(\nu^{(i)}\right) \downarrow_{P_{r}(n) \otimes P_{s}(n)}: L_{r}(\lambda) \otimes L_{s}(\mu)\right] & \text { if } \nu \in \Lambda_{\leq(r+s)}, \\ 0 & \text { otherwise, }\end{cases}
$$

where $\nu=\nu^{(0)} \hookrightarrow_{n} \nu^{(1)} \hookrightarrow_{n} \ldots \hookrightarrow_{n} \nu^{(t)}$ is the $P_{r+s}(n)$-block of $\nu$.

For sufficiently large values of $n$ the partition algebra is semisimple. Therefore Theorem 3.3 reproves the limiting behaviour of tensor products observed by Murnaghan (see Section 1.2). It also offers the following concrete representation theoretic interpretation of the $\bar{g}_{\lambda, \mu}^{\nu}$.

Corollary 3.4. Let $\lambda \vdash r$ and $\mu \vdash s$ and suppose $|\nu| \leq r+s$. Then we have

$$
\bar{g}_{\lambda, \mu}^{\nu}=\left[\Delta_{r+s}(\nu) \downarrow_{P_{r}(n) \otimes P_{s}(n)}: L_{r}(\lambda) \otimes L_{s}(\mu)\right] .
$$

Remark 3.5. We recover the Murnaghan-Littlewood Theorem as follows. Let $\lambda, \mu, \nu$ be partitions and suppose that $|\lambda|+|\mu|=|\nu|$. Then we have that $\Delta_{r+s}(\nu)=\mathbf{S}(\nu)$, $\Delta_{r}(\lambda)=\mathbf{S}(\lambda)$ and $\Delta_{s}(\mu)=\mathbf{S}(\mu)$, and so we have

$$
\bar{g}_{\lambda, \mu}^{\nu}=c_{\lambda, \mu}^{\nu}
$$

Corollary 3.6. We have that $\bar{g}_{\lambda, \mu}^{\nu}=g_{\lambda_{[n]}, \mu_{[n]}}^{\nu_{[n]}}$ if

$$
n \geq \min \left\{|\lambda|+|\mu|+\nu_{1},|\lambda|+|\nu|+\mu_{1},|\nu|+|\mu|+\lambda_{1}\right\} .
$$

Proof. When $n \geq|\lambda|+|\mu|+\nu_{1}$ we have that $\Delta_{r+s}(\nu)=L_{r+s}(\nu)$ by Proposition 2.3 . The result now follows as

$$
g_{\lambda_{[n]}, \mu_{[n]}}^{\nu_{[n]}}=g_{\lambda_{[n]}, \nu_{[n]}}^{\mu_{[n]}}=g_{\nu_{[n]}, \mu_{[n]}}^{\lambda_{[n]}} .
$$

Corollary 3.6 gives a new proof of Brion's bound [Bri93] for the stability of the Kronecker coefficients using the partition algebra.

\subsection{The Kronecker coefficients as a sum of reduced Kronecker coeffi-} cients. In BOR11 a formula is given for writing the Kronecker coefficients as a sum of reduced Kronecker coefficients. We shall now interpret this formula in the Grothendieck group of the partition algebra by showing that it coincides with the formula in Theorem 3.3 .

Let $\nu_{[n]}$ be a partition of $n$. We make the convention that $\nu_{0}=n-|\nu|$ is the 0 th row of $\nu_{[n]}$. For $i \in \mathbb{Z}_{\geq 0}$ define $\nu_{[n]}^{\dagger i}$ to be the partition obtained from $\nu_{[n]}$ by adding 1 to its first $i-1$ rows and erasing its $i$ th row. In particular, we have $\nu_{[n]}^{\dagger 0}=\nu$.

Theorem 3.7 (Theorem 1.1 of BOR11]). Let $\lambda_{[n]}, \mu_{[n]}, \nu_{[n]} \vdash n$. Then

$$
g_{\lambda_{[n]}, \mu[n]}^{\nu_{[n]}}=\sum_{i=0}^{l}(-1)^{i} \bar{g}_{\lambda, \mu}^{\dagger i}
$$

where $l=\ell\left(\lambda_{[n]}\right) \ell\left(\mu_{[n]}\right)-1$. 
Relating this to the partition algebra, we have the following.

Proposition 3.8. Let $\nu_{[n]} \vdash n$ with $\nu \in \Lambda_{\leq r}$ and let $\nu=\nu^{(0)} \hookrightarrow_{n} \nu^{(1)} \hookrightarrow_{n} \ldots$ be the maximal chain of $n$-pairs in $\Lambda_{\leq r}$. Then the partitions

$$
\nu_{[n]}^{\dagger i}=\nu^{(i)}
$$

for all $i \geq 0$.

Proof. The $i=0$ case is clear from the definitions. We proceed by induction. Assume that

$$
\nu_{[n]}^{\dagger i}=\nu^{(i)} .
$$

Then $\left(\nu^{(i)}\right)_{1}=n-|\nu|+1,\left(\nu^{(i)}\right)_{j}=\nu_{j-1}+1$ for $j \leq i$, and $\left(\nu^{(i)}\right)_{j}=\nu_{j}$ for $j>i$. Therefore

$$
\left|\nu^{(i)}\right|=n-|\nu|+1+\sum_{j \neq i} \nu_{j}+i-1=n-\nu_{i}+i .
$$

We have that $\nu^{(i+1)} / \nu^{(i)}$ is a skew partition consisting of a strip in the $(i+1)$ th row. By definition of an $n$-pair the content, $\operatorname{ct}\left(\nu_{i+1}^{(i+1)}\right)$, of the last node is $n-\left|\nu^{(i)}\right|$. Therefore

$$
\operatorname{ct}\left(\nu_{i+1}^{(i+1)}\right):=\nu_{i+1}^{(i+1)}-(i+1)=n-\left(n-\nu_{i}+i\right)=\nu_{i}-i
$$

and $\nu_{i+1}^{(i+1)}=\nu_{i}+1$, and therefore $\nu_{[n]}^{\dagger(i+1)}=\nu^{(i+1)}$.

Remark 3.9. In Theorem 3.3, $t$ is chosen so that $\left|\nu^{(t)}\right| \leq|\lambda|+|\mu|$ and $\left|\nu^{(t+1)}\right|>$ $|\lambda|+|\mu|$. So Theorems 3.3 and 3.7 seem to give a different number of terms in the sum. For example consider

$$
g_{\left(1^{2}\right),\left(1^{2}\right)}^{(2)}=1 \quad g_{\left(1^{2}\right),\left(1^{2}\right)}^{\left(1^{2}\right)}=0 .
$$

These are given as a sum of one, respectively two, terms in Theorem 3.3. both cases have four terms in Theorem 3.7. Now consider

$$
\lambda_{[n]}=\mu_{[n]}=\nu_{[n]}=(10,10,10) ;
$$

then $\ell\left(\lambda_{[n]}\right) \ell\left(\mu_{[n]}\right)=9$. We have $\nu_{[n]}^{\dagger 8}=\left(11^{3}, 1^{5}\right)$ with $\left|\nu_{[n]}^{\dagger 8}\right|=38$. But $r+s=40$, so we have two more terms in Theorem 3.3 corresponding to $\nu^{(9)}=\left(11^{3}, 1^{6}\right)$ and $\nu^{(10)}=\left(11^{3}, 1^{7}\right)$. However, we can show that in fact the two theorems give the same sum.

First assume that $\ell\left(\lambda_{[n]}\right) \ell\left(\mu_{[n]}\right)-1>t$. Then for all $i>t$ we have

$$
\bar{g}_{\lambda, \mu}^{\nu_{[n]}^{\dagger i}}=0
$$

as $\left|\nu_{[n]}^{\dagger i}\right|>|\lambda|+|\mu|$, and so the two sums coincide.

Now assume that $\ell\left(\lambda_{[n]}\right) \ell\left(\mu_{[n]}\right)-1<t$. Then for all $i>\ell\left(\lambda_{[n]}\right) \ell\left(\mu_{[n]}\right)-1$, we have

$$
i \geq \ell\left(\lambda_{[n]}\right) \ell\left(\mu_{[n]}\right) \geq\left|\lambda_{[n]} \cap\left(\mu_{[n]}\right)^{\prime}\right|,
$$

where $\left(\mu_{[n]}\right)^{\prime}$ denotes the conjugate partition of $\mu_{[n]}$. (To see this observe that the Young diagram of $\lambda_{[n]} \cap\left(\mu_{[n]}\right)^{\prime}$ fits in a rectangle of size $\ell\left(\lambda_{[n]}\right) \times \ell\left(\mu_{[n]}\right)$.) Now we have

$$
\ell\left(\nu^{(i)}\right) \geq i \geq\left|\lambda_{[n]} \cap\left(\mu_{[n]}\right)^{\prime}\right| .
$$

But this implies that $\bar{g}_{\lambda, \mu}^{\nu^{(i)}}=0$ by Dvi93. 


\section{The RESTRICTION OF A StANDARd MOdule to A Young SUbAlgebra}

In this section we compute the restriction of a standard module to a Young subalgebra of the partition algebra.

Set $m=r+s$ for some $r, s \geq 1$ and fix $\delta \in \mathbb{C}^{\times}$. We write $P_{r}=P_{r}(\delta), P_{s}=P_{s}(\delta)$ and $P_{m}=P_{m}(\delta)$. We view $P_{r} \otimes P_{s}$ as a subalgebra of $P_{m}$ by mapping each $d \otimes d^{\prime}$, where $d\left(\right.$ resp. $\left.d^{\prime}\right)$ is an $(r, r)$ - (resp. $(s, s)$-) partition diagram, to the $(m, m)$ partition diagram obtained by putting $d$ and $d^{\prime}$ side by side, with $d$ to the left of $d^{\prime}$. When depicting an $(m, m-l)$-partition diagram we will draw a 'wall' separating the first $r$ northern vertices from the last $s$ northern vertices to emphasize the action of $P_{r} \otimes P_{s}$.

We wish to understand the restriction of $\Delta_{m}(\nu)$ to the subalgebra $P_{r} \otimes P_{s}$. Let $\mathfrak{D}_{r}$ denote the diagonal copy of $\mathfrak{S}_{r}$ in $\mathfrak{S}_{r} \times \mathfrak{S}_{r}$. We will need the following lemmas.

Lemma 4.1. Let $\overline{V(2 r, 0)}$ be the subspace of $V(2 r, 0)$ spanned by all $(2 r, 0)$-partition diagrams having precisely $r$ blocks of the form $\{i, j\}$ with $1 \leq i \leq r$ and $r+1 \leq j \leq$ $2 r$. Then $\overline{V(2 r, 0)}$ is a $\mathfrak{S}_{r} \times \mathfrak{S}_{r}$-module and we have

$$
\overline{V(2 r, 0)} \cong \mathbb{C}_{\mathfrak{D}_{\mathfrak{D}_{r}}}^{\mathfrak{S}_{r} \times \mathfrak{S}_{r}} \cong \bigoplus_{\lambda \vdash r} \mathbf{S}(\lambda) \otimes \mathbf{S}(\lambda) .
$$

Proof. A basis for $\overline{V(2 r, 0)}$ is given by the set $\left\{(1, \kappa) v_{0}: \kappa \in \mathfrak{S}_{r}\right\}$, where $v_{0}$ is the diagram in $V(2 r, 0)$, where vertex $i$ is connected to vertex $r+i$ for all $1 \leq i \leq r$ as pictured in Figure 4

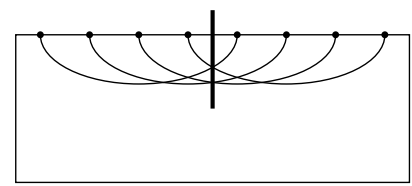

FiguRE 4. The diagram $v_{0}$ in $\overline{V(2 r, 0)}$ (with $r=4$ ).

Now, the map

$$
f: \overline{V(2 r, 0)} \rightarrow \mathbb{C} \uparrow \mathfrak{S}_{\mathfrak{D}_{r}}^{\mathfrak{S}_{r}} \times \mathfrak{S}_{r}=\mathbb{C}\left(\mathfrak{S}_{r} \times \mathfrak{S}_{r}\right) \otimes_{\mathfrak{D}_{r}} \mathbb{C}
$$

given by

$$
f\left((1, \kappa) v_{0}\right)=(1, \kappa) \otimes 1
$$

is clearly a bijection. Moreover, for $\left(\tau, \tau^{\prime}\right) \in \mathfrak{S}_{r} \times \mathfrak{S}_{r}$, we have

$$
\begin{aligned}
f\left(\left(\tau, \tau^{\prime}\right)(1, \kappa) v_{0}\right) & =f\left(\left(\tau, \tau^{\prime} \kappa\right) v_{0}\right) \\
& =f\left(\left(1, \tau^{\prime} \kappa \tau^{-1}\right) v_{0}\right) \quad \text { as shown in Figure [5] } \\
& =\left(1, \tau^{\prime} \kappa \tau^{-1}\right) \otimes 1 \\
& =\left(1, \tau^{\prime} \kappa \tau^{-1}\right) \otimes \tau 1 \\
& =\left(\tau, \tau^{\prime} \kappa\right) \otimes 1 \\
& =\left(\tau, \tau^{\prime}\right)(1, \kappa) \otimes 1 \\
& =\left(\tau, \tau^{\prime}\right) f\left((1, \kappa) v_{0}\right) .
\end{aligned}
$$

Thus $f$ gives the required isomorphism. 


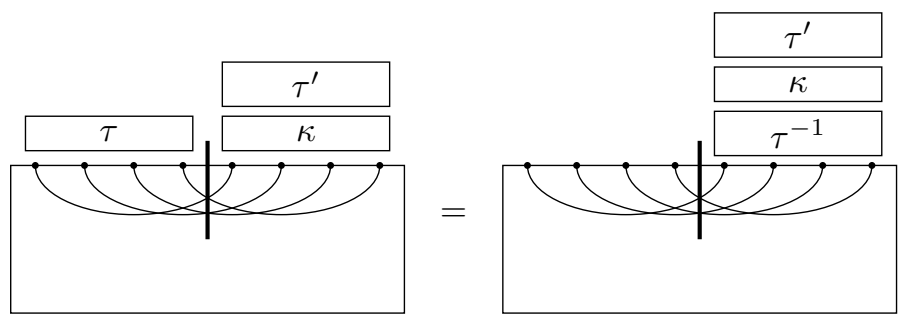

FiguRE 5. The action of $\mathfrak{S}_{r} \times \mathfrak{S}_{r}$ on $(1, \kappa) v_{0}$.

Lemma 4.2. Let $\overline{V(2 r, r)}$ be the subspace of $V(2 r, r)$ spanned by all $(2 r, r)$-partition diagrams having precisely $r$ propagating blocks of the form $\{i, j, \bar{k}\}$ with $1 \leq i \leq r$ and $r+1 \leq j \leq 2 r$. Then, for any $\mu \vdash r$ we have that $\overline{V(2 r, r)} \otimes_{\mathfrak{S}_{r}} \mathbf{S}(\pi)$ is a $\mathfrak{S}_{r} \times \mathfrak{S}_{r}$-module and we have

$$
\overline{V(2 r, r)} \otimes \mathfrak{S}_{r} \mathbf{S}(\pi) \cong \mathbf{S}(\pi) \uparrow_{\mathfrak{D}_{r}}^{\mathfrak{S}_{r} \times \mathfrak{S}_{r}} \cong \bigoplus_{\rho, \sigma \vdash r} g_{\rho, \sigma}^{\pi} \mathbf{S}(\rho) \otimes \mathbf{S}(\sigma)
$$

Proof. Let $X(\pi)$ be a basis for $\mathbf{S}(\pi)$. A basis for $\overline{V(2 r, r)} \otimes_{\mathfrak{S}_{r}} \mathbf{S}(\pi)$ is given by the set

$$
\left\{(1, \kappa) v_{1} \otimes x: \kappa \in \mathfrak{S}_{r}, x \in X(\pi)\right\},
$$

where $v_{1}$ is the diagram in $V(2 r, r)$ with blocks given by $\{i, r+i, \bar{i}\}($ for $1 \leq i \leq r)$ as depicted in Figure 6 .

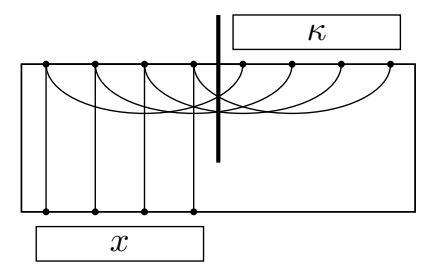

Figure 6 . The element $(1, \kappa) v_{1} \otimes x$.

Now the map

$$
g: \overline{V(2 r, r)} \otimes_{\mathfrak{S}_{r}} \mathbf{S}(\pi) \rightarrow \mathbf{S}(\pi) \uparrow_{\mathfrak{D}_{r}}^{\mathfrak{S}_{r} \times \mathfrak{S}_{r}}=\mathbb{C}\left(\mathfrak{S}_{r} \times \mathfrak{S}_{r}\right) \otimes_{\mathfrak{D}_{r}} \mathbf{S}(\pi)
$$

given by

$$
g\left((1, \kappa) v_{1} \otimes x\right)=(1, \kappa) \otimes x
$$

is clearly a bijection. Moreover, for $\left(\tau, \tau^{\prime}\right) \in \mathfrak{S}_{r} \times \mathfrak{S}_{r}$, we have

$$
\begin{aligned}
g\left(\left(\tau, \tau^{\prime}\right)(1, \kappa) v_{1} \otimes x\right) & =g\left(\left(\tau, \tau^{\prime} \kappa\right) v_{1} \otimes x\right) \\
& =g\left(\left(1, \tau^{\prime} \kappa \tau^{-1}\right) v_{1} \otimes \tau x\right) \quad \text { as shown in Figure } 7 \\
& =\left(1, \tau^{\prime} \kappa \tau^{-1}\right) \otimes \tau x \\
& =\left(\tau, \tau^{\prime} \kappa\right) \otimes x \\
& =\left(\tau, \tau^{\prime}\right)(1, \kappa) \otimes x \\
& =\left(\tau, \tau^{\prime}\right) g\left((1, \kappa) v_{1} \otimes x\right) .
\end{aligned}
$$


Thus $g$ gives the required isomorphism. Finally note that

$$
\begin{aligned}
& \operatorname{dim}_{\mathbb{C}} \operatorname{Hom}_{\mathfrak{S}_{r} \times \mathfrak{S}_{r}}\left(\mathbf{S}(\pi) \uparrow \mathfrak{S}_{\mathfrak{D}_{r}} \times \mathfrak{S}_{r}, \mathbf{S}(\rho) \otimes \mathbf{S}(\sigma)\right) \\
= & \operatorname{dim}_{\mathbb{C}} \operatorname{Hom}_{\mathfrak{D}_{r}}\left(\mathbf{S}(\pi),(\mathbf{S}(\rho) \otimes \mathbf{S}(\sigma)) \downarrow \mathfrak{S}_{\mathfrak{D}_{r}} \times \mathfrak{S}_{r}\right) \\
= & \operatorname{dim}_{\mathbb{C}} \operatorname{Hom}_{\mathfrak{S}_{r}}(\mathbf{S}(\pi), \mathbf{S}(\rho) \otimes \mathbf{S}(\sigma)) \\
= & g_{\rho, \sigma}^{\pi} .
\end{aligned}
$$

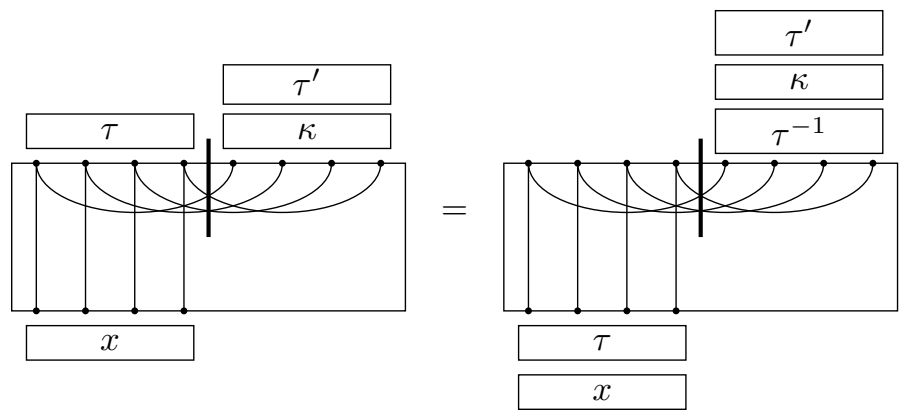

FiguRE 7 . The action of $\mathfrak{S}_{r} \times \mathfrak{S}_{r}$ on $(1, \sigma) v_{1} \otimes x$.

Theorem 4.3. Write $m=r+s$ and let $\nu \vdash m-l, \lambda \vdash r-l_{r}$ and $\mu \vdash s-l_{s}$ for some non-negative integers $l, l_{r}, l_{s}$. Then $\Delta_{m}(\nu) \downarrow_{P_{r} \otimes P_{s}}$ has a filtration by standard modules with multiplicities given by

$$
\left[\Delta_{m}(\nu) \downarrow_{P_{r} \otimes P_{s}}: \Delta_{r}(\lambda) \otimes \Delta_{s}(\mu)\right]=\sum_{\substack{l_{1}, l_{2} \\
l_{1}+2 l_{2}=l-l_{r}-l_{s} \beta \vdash s-l_{s}-l_{1}-l_{2} \\
\pi, \rho, \sigma \vdash l_{1} \\
\gamma \vdash l_{2}}} \sum_{\begin{array}{c}
\alpha \vdash r-l_{2}-l_{1}-l_{2} \\
\alpha, \beta, \pi
\end{array}} c_{\alpha, \rho, \gamma}^{\lambda} c_{\gamma, \sigma, \beta}^{\mu} g_{\rho, \sigma}^{\pi} .
$$

Remark 4.4. Note that these multiplicities are well-defined. This follows by the general theory of quasi-hereditary algebras; see [DR92].

Proof. Recall that $\Delta_{m}(\nu)=V(m, m-l) \otimes_{\mathfrak{S}_{m-l}} \mathbf{S}(\nu)$ and the action of $P_{m}$ on $\Delta_{m}(\nu)$ is given in Section 2.2. Now, we say that a block of a diagram in $V(m, m-l)$ is a crossing block if it contains at least one vertex in $\{1, \ldots, r\}$ and one vertex in $\{r+1, \ldots, r+s\}$. We say that a block of a diagram in $V(m, m-l)$ is a propagating block if it contains at least one vertex in $\{1, \ldots, m\}$ and one vertex in $\{\overline{1}, \ldots, \overline{m-l}\}$. We say that a block is an $r$-propagating block (resp. $s$-propagating block) if it is propagating and it does not contain any vertices in the set $\{r+1, \ldots, r+s\}$ (resp. $\{1, \ldots, r\})$. We claim that there is a filtration of $\Delta_{m}(\nu) \downarrow_{P_{r} \otimes P_{s}}$ with subquotients isomorphic to

$$
V(m, m-l)_{p_{r}, p_{s}, p_{c}, n_{c}} \otimes \mathfrak{S}_{m-l} \mathbf{S}(\nu),
$$

where $V(m, m-l)_{p_{r}, p_{s}, p_{c}, n_{c}}$ denotes the span of all diagrams in $V(m, m-l)$ having precisely $p_{r}$ (resp. $p_{s}$ ) $r$-propagating (resp. $s$-propagating) blocks, $p_{c}$ crossing propagating blocks and $n_{c}$ crossing non-propagating blocks. (Note that we must have $p_{r}+p_{s}+p_{c}=m-l$.) An example is provided in Figure 8 . 


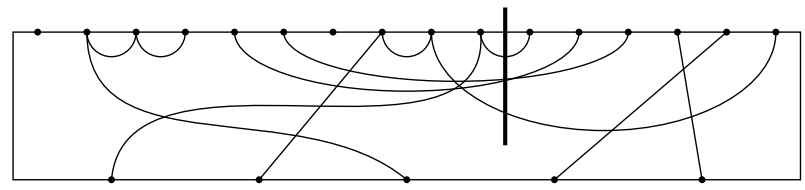

Figure 8. An element of $V(16,5)_{1,2,2,2}$.

To see this, we will consider the action of the generators of $P_{r} \otimes P_{s}$ on $\Delta_{m}(\nu)$. As seen in Section 2.1, $P_{r} \otimes P_{s}$ is generated by the set

$$
\left\{\mathrm{s}_{i, j}, \mathrm{p}_{i, j}: 1 \leq i<j \leq r \text { or } r+1 \leq i<j \leq r+s\right\} \cup\left\{\mathrm{p}_{i}: 1 \leq i \leq r+s\right\} .
$$

Let $v$ be a diagram in $V(m, m-l)_{p_{r}, p_{s}, p_{c}, n_{c}}$, and $X$ be a partition diagram in $P_{r} \otimes P_{s}$. Then we have $X v=\delta^{t} v^{\prime}$ for some $(m, m-l)$-partition diagram $v^{\prime}$ and some nonnegative integer $t$. We write $p_{r}^{\prime}, p_{s}^{\prime}, p_{c}^{\prime}$ and $n_{c}^{\prime}$ for the number of $r$-propagating, $s$-propagating, crossing propagating and crossing non-propagating blocks in $v^{\prime}$, respectively. We will now take for $X$ each of the generators in turn and consider how these numbers relate for $v^{\prime}$ to those for $v$.

If $X=\mathrm{s}_{i, j}$ (for $1 \leq i<j \leq r$ or $r+1 \leq i<j \leq r+s$ ), then it is clear that

$$
p_{r}^{\prime}=p_{r}, p_{s}^{\prime}=p_{s}, p_{c}^{\prime}=p_{c} \text { and } n_{c}^{\prime}=n_{c} .
$$

Now suppose that $X=\mathrm{p}_{i}$ for some $1 \leq i \leq r$ (the case $r+1 \leq i \leq r+s$ is similar, with the role of $r$-propagating and $s$-propagating blocks interchanged). Let $A_{i}$ be the block in $v$ containing vertex $i$. Then all blocks in $v^{\prime}$ are the same as those in $v$, except for $A_{i}$, which is replaced by $A_{i} \backslash\{i\}$ and $\{i\}$. Thus if $A_{i} \backslash\{i\}$ contains a vertex in $\{1,2, \ldots, r\}$ or if $A_{i}$ is a non-propagating non-crossing block, then we have

$$
p_{r}^{\prime}=p_{r}, p_{s}^{\prime}=p_{s}, p_{c}^{\prime}=p_{c} \text { and } n_{c}^{\prime}=n_{c} .
$$

Now suppose that $i$ is the only vertex in $A_{i} \cap\{1, \ldots, r\}$. If $A_{i}$ is a non-propagating crossing block, then we have

$$
p_{r}^{\prime}=p_{r}, p_{s}^{\prime}=p_{s}, p_{c}^{\prime}=p_{c} \text { and } n_{c}^{\prime}=n_{c}-1 .
$$

If $A_{i}$ is a propagating crossing block, then we have

$$
p_{r}^{\prime}=p_{r}, p_{s}^{\prime}=p_{s}+1, p_{c}^{\prime}=p_{c}-1 \text { and } n_{c}^{\prime}=n_{c} .
$$

Finally, if $A_{i}$ is $r$-propagating, then we have $p_{r}^{\prime}=p_{r}-1, p_{s}^{\prime}=p_{s}, p_{c}^{\prime}=p_{c}$ and $n_{c}^{\prime}=n_{c}$, but in this case we have $\mathrm{p}_{i}(v \otimes x)=0$ (where $\left.x \in \mathbf{S}(\nu)\right)$ as $v^{\prime}$ has fewer than $m-l$ propagating blocks (see Section 2.2).

Finally, suppose that $X=\mathrm{p}_{i, j}$ for some $1 \leq i<j \leq r$ (again, the case $r+1 \leq$ $i \leq r+s$ is similar, with the role of $r$-propagating and $s$-propagating interchanged). Denote by $A_{i}$ (resp. $A_{j}$ ) the block containing $i$, resp. $j$. Then we have that the blocks of $v^{\prime}$ are the same as those of $v$ except that the blocks $A_{i}$ and $A_{j}$ are now replaced by a single block $A_{i} \cup A_{j}$. Thus, if $A_{i}=A_{j}$ or if one of $A_{i}$ or $A_{j}$ is a non-propagating non-crossing block, then we have

$$
p_{r}^{\prime}=p_{r}, p_{s}^{\prime}=p_{s}, p_{c}^{\prime}=p_{c} \text { and } n_{c}^{\prime}=n_{c} .
$$

Now suppose that $A_{i} \neq A_{j}$. If both $A_{i}$ and $A_{j}$ are non-propagating crossing blocks, then we have

$$
p_{r}^{\prime}=p_{r}, p_{s}^{\prime}=p_{s}, p_{c}^{\prime}=p_{c} \text { and } n_{c}^{\prime}=n_{c}-1 \text {. }
$$


If one of $A_{i}$ or $A_{j}$ is non-propagating crossing and the other is propagating crossing, then we have

$$
p_{r}^{\prime}=p_{r}, p_{s}^{\prime}=p_{s}, p_{c}^{\prime}=p_{c} \text { and } n_{c}^{\prime}=n_{c}-1 \text {. }
$$

If both $A_{i}$ and $A_{j}$ are propagating crossing blocks, then we have $p_{r}^{\prime}=p_{r}, p_{s}^{\prime}=p_{s}$, $p_{c}^{\prime}=p_{c}-1$ and $n_{c}^{\prime}=n_{c}$, but in this case we get $\mathrm{p}_{i, j}(v \otimes x)=0$ as $v^{\prime}$ has fewer than $m-l$ propagating blocks. If one of $A_{i}$ or $A_{j}$ is $r$-propagating and the other is non-propagating crossing, then we have

$$
p_{r}^{\prime}=p_{r}-1, p_{s}^{\prime}=p_{s}, p_{c}^{\prime}=p_{c}+1 \text { and } n_{c}^{\prime}=n_{c}-1 \text {. }
$$

Finally, if one of $A_{i}$ or $A_{j}$ is $r$-propagating and the other is propagating crossing, then we have $p_{r}^{\prime}=p_{r}-1, p_{s}^{\prime}=p_{s}, p_{c}^{\prime}=p_{c}$ and $n_{c}^{\prime}=n_{c}$, but in this case we get $\mathrm{p}_{i, j}(v \otimes x)=0$ as $v^{\prime}$ has fewer than $m-l$ propagating blocks.

It follows from (4.1)-(4.8) that the number of crossing non-propagating blocks never increases when we apply an element of $P_{r} \otimes P_{s}$, and so we get a filtration of $\Delta_{m}(\nu)$ with subquotients isomorphic to $V(m, m-l)_{n_{c}} \otimes_{\mathfrak{S}_{m-l}} \mathbf{S}(\nu)$, where $V(m, m-l)_{n_{c}}$ is the span of all diagrams in $V(m, m-l)$ having precisely $n_{c}$ crossing non-propagating blocks. Now, we also get from (4.1)-(4.8) that $V(m, m-l)_{n_{c}} \otimes_{\mathfrak{S}_{m-l}} \mathbf{S}(\nu)$ has a filtration by the number of propagating crossing blocks with subquotients isomorphic to $V(m, m-l)_{p_{c}, n_{c}} \otimes_{\mathfrak{S}_{m-l}} \mathbf{S}(\nu)$, where $V(m, m-l)_{p_{c}, n_{c}}$ is the span of all diagrams in $V(m, m-l)_{n_{c}}$ having precisely $p_{c}$ propagating crossing blocks. Moreover, each of these subquotients decomposes further as

$$
\bigoplus_{\substack{p_{r}, p_{s} \\ p_{r}+p_{s}+p_{c}=m-l}} V(m, m-l)_{p_{r}, p_{s}, p_{c}, n_{c}} \otimes_{\mathfrak{S}_{m-l}} \mathbf{S}(\nu) .
$$

This proves the claim.

Now note that as $\left(P_{r} \otimes P_{s}, \mathfrak{S}_{m-l}\right)$-bimodules we have

$$
V(m, m-l)_{p_{r}, p_{s}, p_{c}, n_{c}} \cong V\left(m,\left(p_{r}, p_{c}, p_{s}\right)\right)_{n_{c}} \otimes_{\mathfrak{S}_{p_{r}} \times \mathfrak{S}_{p_{c}} \times \mathfrak{S}_{p_{s}}} \mathbb{C S}_{m-l}
$$

where $V\left(m,\left(p_{r}, p_{c}, p_{s}\right)\right)_{n_{c}}$ is the span of all diagrams in $V(m, m-l)_{p_{r}, p_{s}, p_{c}, n_{c}}$ such that the $r$-propagating blocks connect to the first $p_{r}$ southern vertices, the propagating crossing blocks connect to the next $p_{c}$ southern vertices and the $s$-propagating blocks connect to the last $p_{s}$ southern vertices; see Figure 9 .

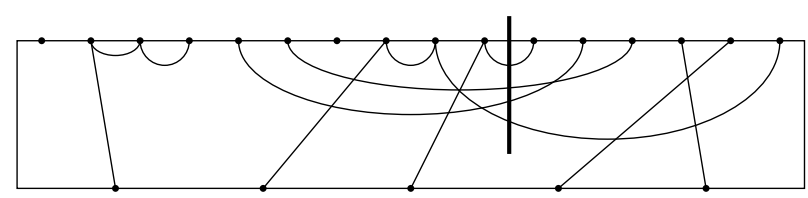

Figure 9. An element, $w$, of $V(16,(1,2,2))_{2}$. 
Thus we get

$$
\begin{aligned}
& V(m, m-l)_{p_{r}, p_{s}, p_{c}, n_{c}} \otimes_{\mathfrak{S}_{m-l}} \mathbf{S}(\nu) \\
\cong & \left(V\left(m,\left(p_{r}, p_{c}, p_{s}\right)\right)_{n_{c}} \otimes_{\mathfrak{S}_{p_{r}} \times \mathfrak{S}_{p_{c}} \times \mathfrak{S}_{p_{s}}} \mathbb{C}_{m-l}\right) \otimes_{\mathfrak{S}_{m-l}} \mathbf{S}(\nu) \\
\cong & V\left(m,\left(p_{r}, p_{c}, p_{s}\right)\right)_{n_{c}} \otimes_{\mathfrak{S}_{p_{r}} \times \mathfrak{S}_{p_{c}} \times \mathfrak{S}_{p_{s}}}\left(\mathbb{C}_{m-l} \otimes_{\mathfrak{S}_{m-l}} \mathbf{S}(\nu)\right) \\
\cong & V\left(m,\left(p_{r}, p_{c}, p_{s}\right)\right)_{n_{c}} \otimes_{\mathfrak{S}_{p_{r}} \times \mathfrak{S}_{p_{c}} \times \mathfrak{S}_{p_{s}}} \mathbf{S}(\nu) \downarrow \mathfrak{S}_{p_{r}} \times \mathfrak{S}_{p_{c}} \times \mathfrak{S}_{p_{s}} \\
\cong & V\left(m,\left(p_{r}, p_{c}, p_{s}\right)\right)_{n_{c}} \otimes_{\mathfrak{S}_{p_{r}} \times \mathfrak{S}_{p_{c}} \times \mathfrak{S}_{p_{s}}} \bigoplus_{\substack{\alpha \vdash p_{r} \\
\beta \vdash p_{s} \\
\pi \vdash p_{c}}}^{\nu} c_{\alpha, \beta, \pi}^{\nu} \mathbf{S}(\alpha) \otimes \mathbf{S}(\pi) \otimes \mathbf{S}(\beta) .
\end{aligned}
$$

From now on we write $\mathfrak{S}_{a_{1}, a_{2}, \ldots, a_{n}}$ for the Young subgroup $\mathfrak{S}_{a_{1}} \times \mathfrak{S}_{a_{2}} \times \ldots \times \mathfrak{S}_{a_{n}}$ of $\mathfrak{S}_{a_{1}+a_{2}+\ldots+a_{n}}$. Now observe that as $\left(P_{r} \otimes P_{s}, \mathfrak{S}_{p_{r}, p_{c}, p_{s}}\right)$-bimodules we have

$$
\begin{aligned}
V\left(m,\left(p_{r}, p_{c}, p_{s}\right)\right)_{n_{c}} & \cong\left(V\left(r, p_{r}+p_{c}+n_{c}\right) \otimes V\left(s, n_{c}+p_{c}+p_{s}\right)\right) \\
& \otimes_{\mathfrak{S}_{p_{r}+p_{c}+n_{c}, n_{c}+p_{c}+p_{s}}} V\left(p_{r}+2 p_{c}+2 n_{c}+p_{s},\left(p_{r}, p_{c}, p_{s}\right)\right)_{n_{c}} .
\end{aligned}
$$

(Note that in $V\left(p_{r}+2 p_{c}+2 n_{c}+p_{s},\left(p_{r}, p_{c}, p_{s}\right)\right)_{n_{c}}$ we place a 'wall' after the first $p_{r}+p_{c}+n_{c}$ northern vertices, and so the triple $\left(p_{r}, p_{c}, p_{s}\right)$ refers to the number of propagating blocks on the left side of the wall, crossing the wall and on the right side of the wall respectively.) This is obtained by decomposing the diagrams as illustrated in Figure 10 .

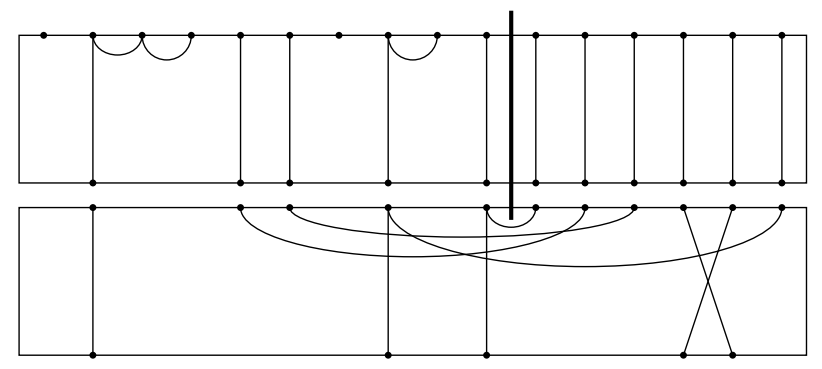

FIGURE 10. Decomposing the diagrams in $V\left(m,\left(p_{r}, p_{c}, p_{s}\right)\right)_{n_{c}}$.

Moreover, as $\left(\mathfrak{S}_{p_{r}+p_{c}+n_{c}, n_{c}+p_{c}+p_{s}}, \mathfrak{S}_{p_{r}, p_{c}, p_{s}}\right)$-bimodules we have

$$
V\left(p_{r}+2 p_{c}+2 n_{c}+p_{s},\left(p_{r}, p_{c}, p_{s}\right)\right)_{n_{c}} \cong \mathbb{C S}_{p_{r}+p_{c}+n_{c}, n_{c}+p_{c}+p_{s}} \otimes \mathfrak{S}_{p_{r}, p_{c}, n_{c}, n_{c}, p_{c}, p_{s}},
$$

$$
\left(V\left(p_{r}, p_{r}\right) \otimes \overline{V\left(2 p_{c}, p_{c}\right)} \otimes \overline{V\left(2 n_{c}, 0\right)} \otimes V\left(p_{s}, p_{s}\right)\right),
$$

where we embed $V\left(p_{r}, p_{r}\right) \otimes \overline{V\left(2 p_{c}, p_{c}\right)} \otimes \overline{V\left(2 n_{c}, 0\right)} \otimes V\left(p_{s}, p_{s}\right) \quad$ inside $V\left(p_{r}+2 p_{c}+2 n_{c}+p_{s},\left(p_{r}, p_{c}, p_{s}\right)\right)_{n_{c}}$ by putting $V\left(p_{r}, p_{r}\right)$ on the set of vertices $\left\{1, \ldots, p_{r}, \overline{1}, \ldots, \overline{p_{r}}\right\}, \overline{V\left(2 p_{c}, p_{c}\right)}$ on the set of vertices $\left\{p_{r}+1, \ldots, p_{r}+p_{c}, p_{r}+p_{c}+\right.$ $\left.2 n_{c}+1, \ldots, p_{r}+2 p_{c}+2 n_{c}, \overline{p_{r}+1}, \ldots, \overline{p_{r}+p_{c}}\right\}, \overline{V\left(2 n_{c}, 0\right)}$ on the set of vertices $\left\{p_{r}+p_{c}+1, \ldots, p_{r}+p_{c}+2 n_{c}\right\}$, and $V\left(p_{s}, p_{s}\right)$ on the remaining vertices. This is illustrated in Figure 11 . 


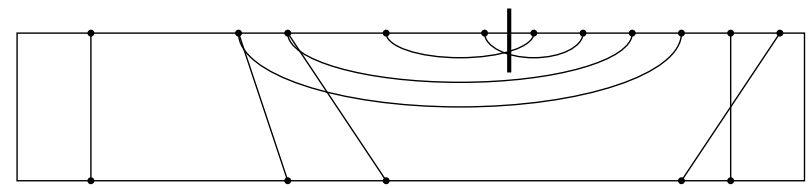

Figure 11. An element of $V\left(p_{r}, p_{r}\right) \otimes \overline{V\left(2 p_{c}, p_{c}\right)} \otimes \overline{V\left(2 n_{c}, 0\right)} \otimes$ $V\left(p_{s}, p_{s}\right)$ embedded into $V\left(p_{r}+2 p_{c}+2 n_{c}+p_{s},\left(p_{r}, p_{c}, p_{s}\right)\right)_{n_{c}}$ with $p_{r}=1$ and $p_{s}=p_{c}=n_{c}=2$.

It follows that

$$
\begin{aligned}
& V\left(p_{r}+2 p_{c}+2 n_{c}+p_{s},\left(p_{r}, p_{c}, p_{s}\right)\right)_{n_{c}} \otimes_{\mathfrak{S}_{p_{r}, p_{c}, p_{s}}}(\mathbf{S}(\alpha) \otimes \mathbf{S}(\pi) \otimes \mathbf{S}(\beta)) \\
\cong \quad & {\left[\left(V\left(p_{r}, p_{r}\right) \otimes \overline{V\left(2 p_{c}, p_{c}\right)} \otimes \overline{V\left(2 n_{c}, 0\right)} \otimes V\left(p_{s}, p_{s}\right)\right) \otimes_{\mathfrak{S}_{p_{r}, p_{c}, p_{s}}}\right.} \\
& (\mathbf{S}(\alpha) \otimes \mathbf{S}(\pi) \otimes \mathbf{S}(\beta)) \uparrow^{\mathfrak{S}_{p_{r}+p_{c}+n_{c}, n_{c}+p_{c}+p_{s}}} \mathfrak{S}_{p_{r}, p_{c}, n_{c}, n_{c}, p_{c}, p_{s}} \\
\cong \quad & {\left[\left(V\left(p_{r}, p_{r}\right) \otimes_{\mathfrak{S}_{p_{r}}} \mathbf{S}(\alpha)\right) \otimes\left(\overline{V\left(2 p_{c}, p_{c}\right)} \otimes_{\mathfrak{S}_{p_{c}}} \mathbf{S}(\pi)\right) \otimes \overline{V\left(2 n_{c}, 0\right)}\right.} \\
& \left.\bigotimes\left(V\left(p_{s}, p_{s}\right) \otimes_{\mathfrak{S}_{p_{s}}} \mathbf{S}(\beta)\right)\right] \uparrow_{\mathfrak{S}_{p_{r}+p_{c}+n_{c}, n_{c}+p_{c}+p_{s}} . n_{c}, n_{c}, p_{c}, p_{s}} .
\end{aligned}
$$

Using Lemmas 4.1 and 4.2 and the fact that $V\left(p_{r}, p_{r}\right) \cong \mathbb{C S}_{p_{r}}$ and similarly for $V\left(p_{s}, p_{s}\right)$ we get that it is isomorphic to

$$
\left(\bigoplus_{\gamma \vdash n_{c}} \bigoplus_{\rho, \sigma \vdash p_{c}} g_{\rho, \sigma}^{\pi} \mathbf{S}(\alpha) \otimes \mathbf{S}(\rho) \otimes \mathbf{S}(\gamma) \otimes \mathbf{S}(\gamma) \otimes \mathbf{S}(\sigma) \otimes \mathbf{S}(\beta)\right) \uparrow \uparrow_{\mathfrak{S}_{p_{r}, p_{c}, n_{c}, n_{c}, p_{c}, p_{s}}}^{\mathfrak{S}_{p_{r}+p_{c}+n_{c}, n_{c}+p_{c}+p_{s}}} .
$$

Now applying the Littlewood-Richardson rule we get that this is isomorphic to

$$
\bigoplus_{\gamma \vdash n_{c} \rho, \sigma \vdash p_{c}} \bigoplus_{\rho, \sigma}^{\pi} \bigoplus_{\substack{\lambda \vdash p_{r}+p_{c}+n_{c} \\ \mu \vdash p_{s}+p_{c}+n_{c}}} c_{\alpha, \rho, \gamma}^{\lambda} c_{\gamma, \sigma, \beta}^{\mu} \mathbf{S}(\lambda) \otimes \mathbf{S}(\mu) .
$$

Combining this with equation (4.10) for a given direct summand in the decomposition (4.9) we obtain

$$
\begin{aligned}
& V\left(m,\left(p_{r}, p_{c}, p_{s}\right)\right)_{n_{c}} \otimes \mathfrak{S}_{p_{r}, p_{c}, p_{s}}(\mathbf{S}(\alpha) \otimes \mathbf{S}(\pi) \otimes \mathbf{S}(\beta)) \\
& \left(V\left(r, p_{r}+p_{c}+n_{c}\right) \otimes V\left(s, n_{c}+p_{c}+p_{s}\right)\right) \otimes \mathfrak{S}_{p_{r}+p_{c}+n_{c}, n_{c}+p_{c}+p_{s}} \\
& \left(\bigoplus_{\gamma \vdash n_{c} \rho, \sigma \vdash p_{c}} g_{\rho, \sigma}^{\pi} \bigoplus_{\substack{\lambda \vdash p_{r}+p_{c}+n_{c} \\
\mu \vdash p_{s}+p_{c}+n_{c}}} c_{\alpha, \rho, \gamma}^{\lambda} c_{\gamma, \sigma, \beta}^{\mu} \mathbf{S}(\lambda) \otimes \mathbf{S}(\mu)\right) \\
\cong & \bigoplus \bigoplus_{\gamma \vdash n_{c} \rho, \sigma \vdash p_{c}} g_{\rho, \sigma}^{\pi} \bigoplus_{\substack{\lambda \vdash p_{r}+p_{c}+n_{c} \\
\mu \vdash p_{s}+p_{c}+n_{c}}} c_{\alpha, \rho, \gamma}^{\lambda} c_{\gamma, \sigma, \beta}^{\mu} \\
& \left(V\left(r, p_{r}+p_{c}+n_{c}\right) \otimes_{\mathfrak{S}_{p_{r}+p_{c}+n_{c}}} \mathbf{S}(\lambda)\right) \otimes\left(V\left(s, n_{c}+p_{c}+p_{s}\right) \otimes_{\mathfrak{S}_{n_{c}+p_{c}+p_{s}}} \mathbf{S}(\mu)\right) .
\end{aligned}
$$

But by the definition of the standard modules $\Delta_{r}(\lambda)$ and $\Delta_{s}(\mu)$, this can be rewritten as

$$
\bigoplus_{\substack{\gamma \vdash n_{c} \\ \rho, \sigma \vdash p_{c} \\ \lambda \vdash p_{r}+p_{c}+p_{c}+n_{c}}} \bigoplus_{\rho, \sigma}^{\pi} c_{\alpha, \rho, \gamma}^{\lambda} c_{\gamma, \sigma, \beta}^{\mu} \Delta_{r}(\lambda) \otimes \Delta_{s}(\mu) .
$$

Now noting that $p_{r}+p_{s}+p_{c}=m-l, p_{r}+p_{c}+n_{c}=r-l_{r}$ and $p_{s}+p_{c}+n_{c}=s-l_{s}$ and writing $l_{1}=p_{c}$ and $l_{2}=n_{c}$, we get that $l-l_{r}-l_{s}=l_{1}+l_{2}$, and the result follows by combining with (4.9). 
In [BOR11, Lemma 2.1] a formula is given for writing the reduced Kronecker coefficients as a sum of Kronecker coefficients and Littlewood-Richardson coefficients. An immediate corollary of the above theorem is an interpretation of this formula in the setting of the partition algebra.

Corollary 4.5. Let $\lambda, \mu, \nu$ be any partitions with $|\lambda|=r,|\mu|=s$ and $|\nu|=r+s-l$. Then the reduced Kronecker coefficient $\bar{g}_{\lambda, \mu}^{\nu}$ is given by

$$
\bar{g}_{\lambda, \mu}^{\nu}=\sum_{\substack{l_{1}, l_{2} \\ l=l_{1}+2 l_{2}}} \sum_{\substack{\alpha \vdash r-l_{1}-l_{2} \\ \beta \vdash s-l_{1}-l_{2}}} \sum_{\substack{\pi, \rho, \sigma \vdash l_{1} \\ \gamma \vdash l_{2}}} c_{\alpha, \beta, \pi}^{\nu} c_{\alpha, \rho, \gamma}^{\lambda} c_{\gamma, \sigma, \beta}^{\mu} g_{\rho, \sigma}^{\pi} .
$$

Proof. This follows from Theorems 3.3 and 4.3, noting that for $|\lambda|=r$ and $|\mu|=s$, $\Delta_{r}(\lambda)=L_{r}(\lambda)$ and $\Delta_{r}(\mu)=L_{r}(\mu)$.

\section{HOOKS AND TWO-PART PARTITIONS}

We now consider the case where one of the partitions in a reduced Kronecker coefficient is either a hook or two-part partition. The first positive formula for the two-part partition case was due to Ballantine and Orellana [BO05]. Blasiak Bla12] has recently given a combinatorial interpretation of the one hook case.

The result below provides positive formulas for $g_{\lambda_{[n]}, \mu_{[n]}}^{\nu_{n]}}$ in the case that $\nu_{[n]}$ is a two-part or hook partition and $n$ is sufficiently large. These formulas reveal a distinct symmetry between the two cases.

Corollary 5.1. Let $\lambda_{[n]}, \mu_{[n]}, \nu_{[n]}$ be partitions of $n$ with $|\lambda|=r,|\mu|=s$ and $|\nu|=r+s-l$.

(i) Suppose $\nu_{[n]}=(n-k, k)$ is a two-part partition. Then we have

$$
g_{\lambda_{[n]}, \mu[n]}^{(n-k, k)}=\bar{g}_{\lambda, \mu}^{(k)}=\sum_{\substack{l_{1}, l_{2} \\ l=l_{1}+2 l_{2}}} \sum_{\substack{\sigma \vdash l_{1} \\ \gamma \vdash l_{2}}} c_{\left(r-l_{1}-l_{2}\right), \sigma, \gamma}^{\lambda} c_{\gamma, \sigma,\left(s-l_{1}-l_{2}\right)}^{\mu}
$$

for all $n \geq \min \left\{|\lambda|+\mu_{1}+k,|\mu|+\lambda_{1}+k\right\}$.

(ii) Suppose $\nu_{[n]}=\left(n-k, 1^{k}\right)$ is a hook partition. Then we have

$$
g_{\lambda_{[n]}, \mu[n]}^{\left(n-k, 1^{k}\right)}=\bar{g}_{\lambda, \mu}^{\left(1^{k}\right)}=\sum_{\substack{l_{1}, l_{2} \\ l=l_{1}+2 l_{2}}} \sum_{\substack{\sigma \vdash l_{1} \\ \gamma \vdash l_{2}}} c_{\left(1^{\left.r-l_{1}-l_{2}\right), \sigma, \gamma}\right.}^{\lambda} c_{\gamma, \sigma^{\prime},\left(1^{\left.s-l_{1}-l_{2}\right)}\right.}^{\mu}
$$

for all $n \geq \min \left\{|\lambda|+|\mu|+1,|\mu|+\lambda_{1}+k,|\lambda|+\mu_{1}+k\right\}$ and where $\sigma^{\prime}$ denotes the transpose of $\sigma$.

Proof. Our assumption on $n$ implies that $g_{\lambda_{[n]}, \mu_{[n]}}^{\nu_{[n]}}=\bar{g}_{\lambda, \mu}^{\nu}$ by Corollary 3.6.

The result follows from Corollary 4.5 noting that $c_{\alpha, \beta, \pi}^{(k)}$ (respectively $c_{\alpha, \beta, \pi}^{\left(1^{k}\right)}$ ) is zero unless $\alpha=\left(r-l_{1}-l_{2}\right), \beta=\left(s-l_{1}-l_{2}\right), \pi=\left(l_{1}\right)$ (respectively $\alpha=\left(1^{r-l_{1}-l_{2}}\right)$, $\left.\beta=\left(1^{s-l_{1}-l_{2}}\right), \pi=\left(1^{l_{1}}\right)\right)$, in which case it is equal to 1 and $g_{\rho, \sigma}^{\left(l_{1}\right)}$ (respectively $g_{\rho, \sigma}^{\left(1^{l_{1}}\right)}$ ) is zero unless $\rho=\sigma$ (respectively $\rho=\sigma^{\prime}$ ), in which case it is equal to 1 .

Remark 5.2. In BO05] they compute the Kronecker coefficients

$$
g_{(n-k, k), \lambda_{[n]}}^{\mu_{[n]}}=g_{\lambda_{[n]}, \mu_{[n]}}^{(n-k, k)}
$$

when $n-|\lambda|-\lambda_{1} \geq 2 k$, equivalently

$$
n \geq|\lambda|+\lambda_{1}+2 k \text {. }
$$


Noting that $k=|\mu|$ and for $\bar{g}_{\lambda, \mu}^{\nu} \neq 0$ we must have that $|\mu| \leq|\lambda|+|\nu|$, we see that Corollary 5.1 improves this bound (as $|\mu|+\lambda_{1}+k \leq|\lambda|+\lambda_{1}+2 k$ ).

\section{EXAMPLE}

In this section, we shall compute the tensor square of the Specht module, $\mathbf{S}(n-1,1)$ for $n \geq 2$, labelled by the first non-trivial hook, via the partition algebra. We have that

$\operatorname{Hom}_{\mathfrak{S}_{n}}\left(\mathbf{S}\left(\nu_{[n]}\right), \mathbf{S}(n-1,1) \otimes \mathbf{S}(n-1,1)\right) \cong \operatorname{Hom}_{P_{1}(n) \otimes P_{1}(n)}\left(L_{1}(1) \otimes L_{1}(1), L_{2}(\nu) \downarrow\right)$ if $\nu \in \Lambda_{\leq 2}$ and zero otherwise. Therefore, it is enough to consider the restriction of simple modules from $P_{2}(n)$ to the Young subalgebra $P_{1}(n) \otimes P_{1}(n)$.

The partition algebra $P_{2}(n)$ is a 15-dimensional algebra with basis:
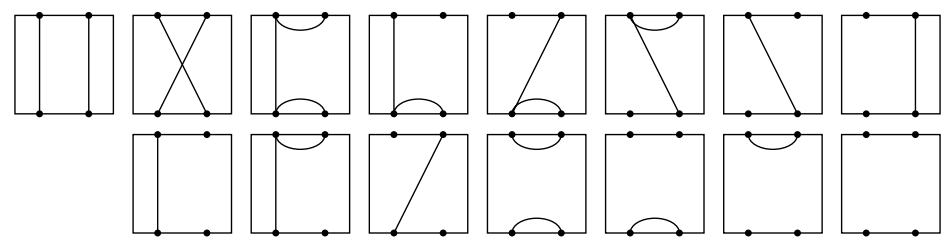

and the product of $x, y \in P_{2}(n)$ is defined by concatenation of $x$ above $y$. For example:
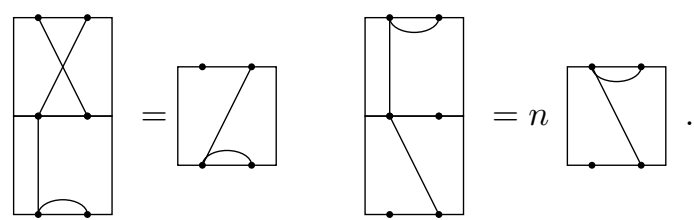

There are four standard modules corresponding to the partitions of degree less than or equal to 2; these are obtained by inflating the Specht modules from the symmetric groups of degree $0,1,2$. These modules have bases:

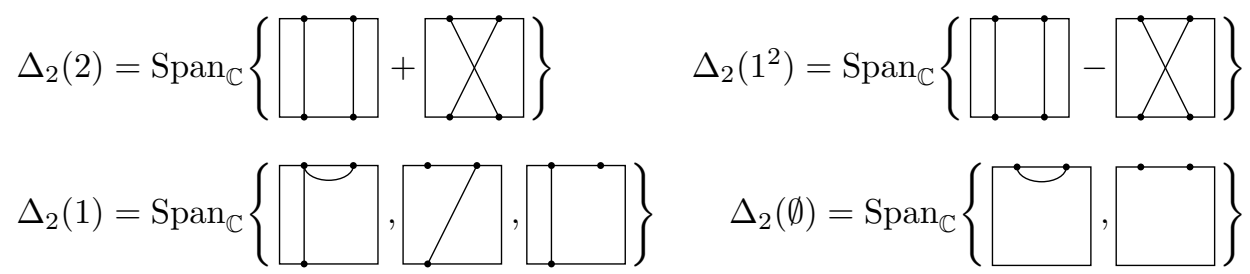

The left-action of $P_{2}(n)$ on the standard modules is given by concatenation on the top. If the resulting diagram has fewer propagating lines than the original, we set the product equal to zero.

The algebra $P_{1}(n) \otimes P_{1}(n)$ is the 4-dimensional subalgebra of $P_{2}(n)$ spanned by the diagrams with no lines crossing an imagined vertical wall down the centre of the diagram.

The restriction of the standard modules to this subalgebra is as follows:

$$
\begin{gathered}
\Delta_{2}(2) \downarrow_{P_{1}(n) \otimes P_{1}(n)} \cong \Delta_{1}(1) \otimes \Delta_{1}(1), \quad \Delta_{2}\left(1^{2}\right) \downarrow_{P_{1}(n) \otimes P_{1}(n)} \cong \Delta_{1}(1) \otimes \Delta_{1}(1), \\
\Delta_{2}(1) \downarrow_{P_{1}(n) \otimes P_{1}(n)} \cong \Delta_{1}(1) \otimes \Delta_{1}(1) \oplus \Delta_{1}(\emptyset) \otimes \Delta_{1}(1) \oplus \Delta_{1}(1) \otimes \Delta_{1}(\emptyset), \\
\Delta_{2}(\emptyset) \downarrow_{P_{1}(n) \otimes P_{1}(n)} \cong \Delta_{1}(1) \otimes \Delta_{1}(1) \oplus \Delta_{1}(\emptyset) \otimes \Delta_{1}(\emptyset) .
\end{gathered}
$$

In particular, note that $\bar{g}_{(1),(1)}^{\nu}=\left[\Delta_{2}(\nu) \downarrow_{P_{1}(n) \otimes P_{1}(n)}: \Delta_{1}(1) \otimes \Delta_{1}(1)\right]=1$ for $\nu=\emptyset, 1,1^{2}, 2$. 
The partition algebra $P_{2}(n)$ is semisimple for $n>2$. For $\nu=\emptyset,(1),\left(1^{2}\right)$ or $(2)$ we have that $\nu_{[n]}=(n),(n-1,1),\left(n-2,1^{2}\right)$, or $(n-2,2)$ and $\nu_{[n]}$ is a partition for $n \geq 0,2,3,4$ respectively. Therefore the Kronecker coefficients

$$
g_{(n-1,1),(n-1,1)}^{\nu_{[n]}}
$$

stabilise for $n \geq 4$ and are non-zero for $n \geq 4$ if and only if $\nu_{[n]}$ is one of the partitions above.

Now consider the case $n=2$. Neither $\nu=\left(1^{2}\right)$ nor $(2)$ correspond to partitions of 2 ; we therefore consider $\nu=\emptyset$ and (1). We have that $(1) \subset(2)$ is the unique 2-pair of partitions of degree less than or equal to 2 (see Section 3.3). Therefore the only standard $P_{2}(2)$-module which is not simple is $\Delta_{2}(1)$ and we have an exact sequence

$$
0 \rightarrow L_{2}(2) \rightarrow \Delta_{2}(1) \rightarrow L_{2}(1) \rightarrow 0 .
$$

Thus in the Grothendieck group we have that $\left[L_{2}(1)\right]=\left[\Delta_{2}(1)\right]-\left[\Delta_{2}(2)\right]$. Hence, we have that $\left[L_{2}(1) \downarrow_{P_{1}(2) \otimes P_{1}(2)}: L_{1}(1) \otimes L_{1}(1)\right]=0$. We conclude that $g_{\left(1^{2}\right),\left(1^{2}\right)}^{\left(1^{2}\right)}=0$ and $g_{\left(1^{2}\right),\left(1^{2}\right)}^{(2)}=1$, as expected.

\section{ACKNOWLEDGEMEnts}

The authors wish to thank David Speyer for pointing out a crucial error in an earlier version of Theorem 4.3 and Corollary 4.5. We would also like to thank the referee for the helpful suggestions. The second and third authors thank Georgia Benkart, Monica Vazirani and Stephanie van Willigenburg and the Banff International Research Station for providing support and a stimulating environment during the Algebraic Combinatorixx workshop where this project started. The first and third authors are grateful for the financial support received from the ANR and NSF grants ANR-10-BLAN-0110 and DMS-1101740, respectively.

\section{REFERENCES}

[Ben98] D. J. Benson, Representations and cohomology, Cambridge studies in advanced mathematics, vol. I, Cambridge University Press, 1998. MR.1644252

[BK99] C. Bessenrodt and A. Kleshchev, On Kronecker products of complex representations of the symmetric and alternating groups, Pacific J. Math. 190 (1999), no. 2, 201-223, DOI 10.2140/pjm.1999.190.201. MR.1722888 (2000i:20017)

[Bla12] J. Blasiak, Kronecker coefficients of one hook shape, arxiv:1209.2209v2, 2012.

[BO05] C. M. Ballantine and R. C. Orellana, On the Kronecker product $s_{(n-p, p)} * s_{\lambda}$, Electron. J. Combin. 12 (2005), Research Paper 28, 26 pp. (electronic). MR2156682 (2006g:05227)

[BOR11] Emmanuel Briand, Rosa Orellana, and Mercedes Rosas, The stability of the Kronecker product of Schur functions, J. Algebra 331 (2011), 11-27, DOI 10.1016/j.jalgebra.2010.12.026. MR2774644 (2012e:05405)

[Bri93] Michel Brion, Stable properties of plethysm: on two conjectures of Foulkes, Manuscripta Math. 80 (1993), no. 4, 347-371, DOI 10.1007/BF03026558. MR1243152 (95c:20056)

[DR92] Vlastimil Dlab and Claus Michael Ringel, The module theoretical approach to quasihereditary algebras, Representations of algebras and related topics (Kyoto, 1990), London Math. Soc. Lecture Note Ser., vol. 168, Cambridge Univ. Press, Cambridge, 1992, pp. 200-224. MR.1211481 (94f:16026)

[Dvi93] Yoav Dvir, On the Kronecker product of $S_{n}$ characters, J. Algebra 154 (1993), no. 1, 125-140, DOI 10.1006/jabr.1993.1008. MR.1201916 (94a:20023)

[GR85] A. M. Garsia and J. Remmel, Shuffles of permutations and the Kronecker product, Graphs Combin. 1 (1985), no. 3, 217-263, DOI 10.1007/BF02582950. MR951014 (89h:05006) 
[GW98] Roe Goodman and Nolan R. Wallach, Representations and invariants of the classical groups, Encyclopedia of Mathematics and its Applications, vol. 68, Cambridge University Press, Cambridge, 1998. MR:1606831 (99b:20073)

[HR05] Tom Halverson and Arun Ram, Partition algebras, European J. Combin. 26 (2005), no. 6, 869-921, DOI 10.1016/j.ejc.2004.06.005. MR2143201(2006g:05228)

[JK81] Gordon James and Adalbert Kerber, The representation theory of the symmetric group, Encyclopedia of Mathematics and its Applications, vol. 16, Addison-Wesley Publishing Co., Reading, Mass., 1981. With a foreword by P. M. Cohn; with an introduction by Gilbert de B. Robinson. MR644144 (83k:20003)

[Jon94] V. F. R. Jones, The Potts model and the symmetric group, Subfactors (Kyuzeso, 1993), World Sci. Publ., River Edge, NJ, 1994, pp. 259-267. MR.1317365 (97b:82023)

[Kly04] A. Klyachko, Quantum marginal problem and representations of the symmetric group, arXiv:quant-ph/0409113 (2004).

[Las80] A. Lascoux, Produit de Kronecker des représentations du groupe symétrique (French), année (Paris, 1979), Lecture Notes in Math., vol. 795, Springer, Berlin, 1980, pp. 319329. MR582085 (82b:20016)

[Lit58] D. E. Littlewood, Products and plethysms of characters with orthogonal, symplectic and symmetric groups, Canad. J. Math. 10 (1958), 17-32. MR0095209 (20 \#1715)

[Mac95] I. G. Macdonald, Symmetric functions and Hall polynomials, 2nd ed., Oxford Mathematical Monographs, The Clarendon Press Oxford University Press, New York, 1995. With contributions by A. Zelevinsky; Oxford Science Publications. MR.1354144 (96h:05207)

[Mar91] Paul Martin, Potts models and related problems in statistical mechanics, Series on Advances in Statistical Mechanics, vol. 5, World Scientific Publishing Co. Inc., Teaneck, NJ, 1991. MR:1103994 (92m:82030)

[Mar96] Paul Martin, The structure of the partition algebras, J. Algebra 183 (1996), no. 2, 319-358, DOI 10.1006/jabr.1996.0223. MR1399030 (98g:05152)

[MS93] Paul Martin and Hubert Saleur, On an algebraic approach to higher-dimensional statistical mechanics, Comm. Math. Phys. 158 (1993), no. 1, 155-190. MR.1243720(94k:82037)

[Mur38] F. D. Murnaghan, The Analysis of the Kronecker Product of Irreducible Representations of the Symmetric Group, Amer. J. Math. 60 (1938), no. 3, 761-784, DOI 10.2307/2371610. MR 1507347

[Mur55] Francis D. Murnaghan, On the analysis of the Kronecker product of irreducible representations of $S_{n}$, Proc. Nat. Acad. Sci. U.S.A. 41 (1955), 515-518. MR0070640 (17,12b)

[Thi91] Jean-Yves Thibon, Hopf algebras of symmetric functions and tensor products of symmetric group representations, Internat. J. Algebra Comput. 1 (1991), no. 2, 207-221, DOI 10.1142/S0218196791000134. MR1128013 (92m:05201)

[Val99] Ernesto Vallejo, Stability of Kronecker products of irreducible characters of the symmetric group, Electron. J. Combin. 6 (1999), Research Paper 39, 7 pp. (electronic). MR:1725703(2001b:20022)

Institut de Mathématiques de Jussieu, 5 Rue du Thomas Mann, 75013, Paris, France E-mail address: Bowman@math.jussieu.fr

Centre for Mathematical Science, City University London, Northampton Square, LONDON, EC1V 0HB, ENGLAND

E-mail address: Maud.Devisscher.1@city.ac.uk

Department of Mathematics, 6188 Kemeny Hall, Dartmouth College, Hanover, New HAMPSHIRE 03755

E-mail address: Rosa.C.Orellana@dartmouth.edu 